\title{
TRIAGEM AUDITIVA NEONATAL EM HOSPITAL DA REDE CEGONHA
}

\author{
Neonatal hearing screening in the Rede Cegonha hospital \\ Tamizaje auditivo neonatal de un hospital de la Rede Cegonha
}

\author{
Luíza Silva Vernier iD \\ Universidade Federal de Ciências da Saúde de Porto Alegre - UFCSPA - Porto Alegre (RS) - Brasil \\ Carla Thamires Rodriguez Castelli iD \\ Universidade Federal de Ciências da Saúde de Porto Alegre - UFCSPA - Porto Alegre (RS) - Brasil
}

Shayenne Silveira Rothermel iD

Universidade Federal de Ciências da Saúde de Porto Alegre - UFCSPA - Porto Alegre (RS) - Brasil

Tatiana de Carvalho Paniz iD

Hospital Materno-Infantil Presidente Vargas - HMIPV - Porto Alegre (RS) - Brasil

Claudia Zanini iD

Hospital Materno-Infantil Presidente Vargas - HMIPV - Porto Alegre (RS) - Brasil

Daniela Centenaro Levandowski iD

Universidade Federal de Ciências da Saúde de Porto Alegre - UFCSPA - Porto Alegre (RS) - Brasil

\section{RESUMO}

Objetivo: Avaliar o perfil de neonatos que realizaram triagem auditiva neonatal (TAN) e suas mães em um hospital público vinculado à Rede Cegonha, no que diz respeito às características sociodemográficas e aos desfechos de saúde. Métodos: Estudo transversal, retrospectivo e documental, baseado em dados de prontuários de um hospital materno-infantil da cidade de Porto Alegre, Rio Grande do Sul. A amostra foi composta por 1.818 díades mãe-bebê, que realizaram TAN no período de maio/2014 a maio/2015. Foram analisados os resultados da TAN e as características clínicas maternas e dos neonatos. Para a análise dos dados, utilizou-se o programa estatístico SPSS, versão 21.0. Resultados: Dos 1.818 neonatos que realizaram a TAN, 359 (19,74\%) tinham algum indicador de risco para deficiência auditiva. Houve associação entre falha dos neonatos na TAN e indicadores de risco para deficiência auditiva (presença de HIV congênito, $p=0,035$; citomegalovírus congênito, $p=0,048$; e exposição a drogas ototóxicas, $p=0,011$ ) e entre ausência de algum indicador de risco para deficiência auditiva com disfunção respiratória $(p=0,043)$ e sepse neonatal $(p=0,021)$. Nos neonatos sem esses indicadores, essa associação aconteceu naqueles cujas mães tiveram infecção de trato urinário na gestação $(p=0,015)$. Conclusão: Verificaram-se associações significativas entre falha na TAN e características maternas e do bebê que não são consideradas como indicadores de risco para deficiência auditiva.

Descritores: Serviços de Saúde Materno-Infantil; Fonoaudiologia; Triagem Neonatal; Audição.

\section{ABSTRACT}

Objective: To evaluate the profile of neonates who underwent neonatal hearing screening (NHS) and their mothers in a public hospital linked to the Rede Cegonha program, concerning sociodemographic characteristics and health outcomes. Methods: Cross-sectional, retrospective and documentary study, based on data from medical records of a maternal and child hospital in the city of Porto Alegre, Rio Grande do Sul. The sample consisted of 1,818 mother-baby dyads, who performed NHS from May 2014 to May 2015. The results of the NHS and maternal and neonatal clinical characteristics were analyzed. For data analysis, the statistical program SPSS version 21.0 was used. Results: Of the 1,818 neonates who underwent NHS, 359 (19.74\%) had some risk indicator for hearing loss. Neonatal failure in NHS was associated with risk indicators for hearing impairment (presence of congenital HIV, $p=0.035$; congenital cytomegalovirus, $p=0.048$; and exposure to ototoxic drugs, $p=0.011$ ) and between absence of any risk indicator for hearing impairment with respiratory dysfunction $(p=0.043)$ and neonatal sepsis $(p=0.021)$. In neonates without these indicators, this association occurred in those whose mothers had urinary tract infection during pregnancy

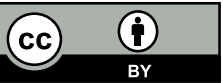


( $p=0.015)$. Conclusion: Significant associations were found between NHS failure and maternal and infant characteristics that are not considered as risk indicators for hearing impairment.

Descriptors: Maternal-Child Health Services; Speech, Language and Hearing Sciences; Neonatal Screening; Hearing.

\section{RESUMEN}

Objetivo: Evaluar el perfil de neonatos que han realizado el tamizaje auditivo neonatal (TAN) y sus madres de un hospital público vinculado al programa Rede Cegonha respecto las características y los resultados en la salud. Métodos: Estudio transversal, retrospectivo y documental basado en los datos de historiales clínicos de un hospital materno-infantil de la ciudad de Porto Alegre, Rio Grande de Sur. La muestra ha sido de 1.818 díadas madre-bebé que han realizado el TAN en el período entre mayo/2014 y mayo/2015. Se han analizados los resultados del TAN y las características clínicas maternas y de los neonatos. Se utilizó el programa estadístico SPSS en la versión 21.0 para el análisis de los datos. Resultados: De los 1.818 neonatos que han realizado el TAN, 359 (19,74\%) tenían algún factor de riesgo para la discapacidad auditiva. Hubo asociación entre la falla de los neonatos del TAN y los indicadores de riesgo para la discapacidad auditiva (presencia del HIV congénito, $p=0,035$; citomegalovírus congénito, $p=0,048$; y la exposición para drogas ototoxicas, $p=0,011$ ) y entre la ausencia de algún indicador de riesgo para la discapacidad auditiva con disfunción respiratoria. $(p=0,043)$ y sepsis neonatal $(p=0,021)$. En los neonatos sin eses indicadores, la asociación se dio en aquellos cuyas madres tuvieron infección del trato urinario en la gestación $(p=0,015)$. Conclusión: Se verificaron asociaciones significativas entre la falla del TAN y las características maternas y del bebé que no son consideradas como indicadores de riesgo para la discapacidad auditiva.

Descriptores: Servicios de Salud Materno-Infantil; Fonoaudiología; Tamizaje Neonatal; Audición.

\section{INTRODUÇÃO}

A Rede Cegonha $(\mathrm{RC})$ é uma estratégia do Ministério da Saúde, instituída no âmbito do Sistema Único de Saúde (SUS), que está em vigor no Brasil desde 2011, normatizada pela Portaria n. ${ }^{\circ}$ 1.459. Um dos seus objetivos é fomentar a implementação de um novo modelo de atenção à saúde da mulher e da criança, com foco na atenção ao parto, ao nascimento, ao crescimento e ao desenvolvimento infantil. Essa estratégia assegura aos bebês o direito ao nascimento seguro e humanizado, ao crescimento e ao desenvolvimento saudáveis, com acompanhamento até os vinte e quatro meses de idade. Para atender essa população com qualidade, a RC organiza-se em quatro subdivisões: pré-natal, parto e nascimento; puerpério e atenção integral à saúde da criança; e, o sistema logístico: transporte sanitário e regulação(1).

Os hospitais pertencentes à $\mathrm{RC}$ encaminham um relatório mensal, no qual constam diversos dados de saúde, dentre eles, os referentes à área da Fonoaudiologia: a atuação no aleitamento materno e na triagem auditiva neonatal (TAN). Esse profissional é capacitado para atuar em alojamento conjunto (AC) e unidades de terapia intensiva (UTI) neonatal, perpassando pela avaliação, terapia, acompanhamento, encaminhamento para avaliações, ou mesmo para atendimento de outros profissionais ${ }^{(2)}$.

A TAN é fundamental para a detecção precoce de anormalidades relacionadas à audição dos recém-nascidos. Desde agosto de 2010, tornou-se obrigatória a realização do exame das emissões otoacústicas evocadas (EOA) em todas as crianças nascidas em território nacional pela Lei n. ${ }^{0} 12.303 / 2010^{(3)}$. Nesse exame, mostram-se evidências diretas do mecanismo coclear ativo e sua ausência está relacionada, na maioria dos casos, com a perda auditiva sensorioneural( ${ }^{(4)}$. A TAN tem como resultados "passa" ou "falha". Quando o desfecho é "falha", o teste deve ser realizado novamente. Persistindo a "falha", indica-se a realização do teste potencial evocado auditivo de tronco encefálico (PEATE - automático ou em modo triagem) ${ }^{(5)}$. O PEATE é composto por uma série de ondas, captadas por eletrodos de superfície posicionados na superfície craniana, gerando respostas neurais e apontando a integridade coclear. Esse exame também compreende uma medida eletrofisiológica do oitavo nervo e da função auditiva baixa do tronco encefálico(4).

Para os neonatos e lactentes com indicadores de risco para a deficiência auditiva (IRDA), a escolha já recai automaticamente no PEATE, automático ou em modo triagem ${ }^{(5)}$. Segundo o Ministério da Saúde ${ }^{(5)}$, baseado no Joint committee on infant hearing $(2007)^{(6)}$ e no Comitê multiprofissional em saúde auditiva(2010)(7), dentre os IRDA estão: hereditariedade; exposição a drogas ototóxicas, como antibióticos aminoglicosídeos e/ou diuréticos de alça; hiperbilirrubinemia; anóxia perinatal grave; Apgar neonatal de 0 a 4 no primeiro minuto, ou 0 a 6 no quinto minuto; exposição a infecções congênitas; crianças com má formações craniofaciais envolvendo orelha e osso temporal; portadoras de síndromes genéticas que expressam deficiência auditiva; e aqueles que sofreram infecções bacterianas ou virais pós-natais. 
A TAN deve ser realizada, preferencialmente, nos primeiros dias de vida ( 24 horas a 48 horas após o nascimento), na maternidade, e, no máximo, durante o primeiro mês de vida, a não ser em casos nos quais a saúde da criança não permita a sua realização ${ }^{(6,7)}$.

No que se refere ao estado do Rio Grande do Sul, apenas quatro hospitais foram contemplados com equipamentos para realizar a TAN por meio da política da RC, sendo que, em um destes, se realizou o presente estudo. Diante disso, fica evidenciada a atuação do profissional da Fonoaudiologia voltada para a realização da TAN, em serviços de saúde nos quais a estratégia da RC está implantada. Esse conhecimento poderá qualificar a assistência já prestada no âmbito do programa, ao possibilitar a elaboração de novas ações e estratégias de atenção por todos os profissionais que integram a equipe de assistência.

O presente estudo objetiva avaliar o perfil de neonatos que realizaram triagem auditiva neonatal (TAN) e suas mães, em um hospital público vinculado à Rede Cegonha, no que diz respeito às características sociodemográficas e aos desfechos de saúde.

\section{MÉTODOS}

Trata-se de um estudo transversal, retrospectivo e documental, realizado em um hospital materno-infantil na cidade de Porto Alegre, Rio Grande do Sul, Brasil, que está inserido na Estratégia da Rede Cegonha (RC). A amostra foi composta por 1.808 díades mãe-bebê que estiveram internadas no AC e/ou na UTI Neonatal de maio de 2014 a maio de 2015, e que receberam atendimento fonoaudiológico. Os dados dos prontuários foram coletados em 2016.

Consideraram-se, no presente estudo, todos os prontuários de bebês que realizaram a TAN, no AC e/ou UTI Neonatal, e de suas mães, que continham registro dos resultados da TAN. Excluíram-se os participantes que não apresentavam registro da TAN completo, ou aqueles cujo prontuário estava incompleto.

Coletaram-se as informações a partir dos prontuários e demais registros do hospital, o que não exigiu a utilização de um Termo de Consentimento Livre e Esclarecido. Solicitou-se, entretanto, a anuência do hospital para a realização do estudo, sendo assinado um termo de confidencialidade frente às informações acessadas. Analisaram-se dados referentes à mãe, considerando período gestacional, idade gestacional, via de parto e patologias que ocorreram durante e/ou previamente à gestação. Analisaram-se, também, dados do bebê, quais sejam: peso, resultado do Apgar no primeiro e quinto minuto, sexo, intercorrências, local da internação (AC/UTI Neonatal) e resultado da TAN, além da presença de IRDA.

Para a análise dos dados, utilizou-se o programa estatístico SPSS, versão 21.0. Descreveram-se os dados contínuos em média e desvio padrão; e os dados categóricos em frequência absoluta (n) e percentual. A comparação entre as variáveis categóricas foi analisada por meio do teste qui-quadrado de Pearson. Adotou-se nível de significância de $5 \%$.

Este estudo obteve a aprovação do Comitê de Ética em Pesquisa da Universidade Federal de Ciências da Saúde de Porto Alegre (UFCSPA; Parecer n. ${ }^{\circ}$ 1.332.958) e do Comitê de Ética do Hospital Materno-Infantil Presidente Vargas (Parecer n. ${ }^{\circ}$ 1.387.303). A pesquisa obedeceu às diretrizes da Resolução $n{ }^{\circ} 466 / 12$ do Conselho Nacional de Saúde, a qual regulamenta as pesquisas envolvendo seres humanos no país ${ }^{(8)}$.

\section{RESULTADOS}

Dentre as 1.818 díades participantes do estudo, 918 bebês (50,5\%) eram do sexo masculino e 1.468 (80,74\%) estavam internados no AC. A média de peso dos recém-nascidos foi de 3191,89 gramas, apresentando grande variação (570 a 4935 gramas). A idade gestacional média foi de $38 \pm 8$ semanas. Já o Apgar médio no primeiro minuto foi de 8,06 e, no quinto minuto, de 9,20. Dentre os participantes, observou-se a presença de, pelo menos, um indicador de risco em 359 díades $(19,74 \%)$, sendo que 228 bebês $(63,50 \%)$ desse grupo estavam internados na UTI Neonatal. Já entre os neonatos sem IRDA ( $n=1459 ; 80,25 \%), 1.337(91,63 \%)$ estavam internados no AC. Observou-se prevalência de parto normal entre as díades avaliadas ( $n=1168 ; 64,2 \%$ ). Na Tabela I, encontram-se informações detalhadas das díades conforme a presença ou não de IRDA nos bebês.

Na Figura 1, pode-se observar o resultado da TAN dos recém-nascidos na presença ou ausência de IRDA e conforme o tipo de teste de avaliação aplicado (EOA ou PEATE).

Pode-se observar no fluxograma (Figura 1) que a maior parte dos neonatos avaliados no período descrito tratase de neonatos sem IRDA, totalizando 80,25\% (1.459) da amostra. Destes, 51 (3,49\%) foram encaminhados para reteste após realização do PEATE. Entre os neonatos com IRDA, houve $46(12,8 \%)$ dos neonatos encaminhados para reteste após a realização do PEATE. 
Tabela I - Indicadores de risco para deficiência auditiva e características de neonatos que realizaram a triagem auditiva neonatal entre maio de 2014 e maio de 2015 no Hospital Materno-Infantil Presidente Vargas, Rio Grande do Sul, Brasil, 2016 ( $n=1.818)$.

\begin{tabular}{|c|c|c|c|}
\hline & Neonatos com IRDA & Neonatos sem IRDA & Total de neonatos (com e sem IRDA) \\
\hline \multicolumn{4}{|l|}{ Via de parto - $n(\%)$} \\
\hline Parto Normal & $192(53,5)$ & $976(66,9)$ & $1168(64,2)$ \\
\hline Parto Cesáreo & $167(46,5)$ & $483(33,1)$ & $650(35,8)$ \\
\hline \multicolumn{4}{|l|}{ Sexo - $n(\%)$} \\
\hline Feminino & $159(44,3)$ & $741(50,8)$ & $900(49,5)$ \\
\hline Masculino & $200(55,7)$ & $718(49,2)$ & $918(50,5)$ \\
\hline \multicolumn{4}{|l|}{ Peso (gramas) } \\
\hline Média (DP) & $2848,09(865,03)$ & $3276,60(504,26)$ & $3191,89(617,02)$ \\
\hline Mínimo-Máximo & $570-4815$ & $1190-4935$ & $570-4935$ \\
\hline \multicolumn{4}{|c|}{ Idade Gestacional (semanas) } \\
\hline Média (DP) & $37 \pm 6(3,17)$ & $39 \pm 1(1,48)$ & $38 \pm 8(2,02)$ \\
\hline Mínimo-Máximo & $26-42 \pm 4$ & $25 \pm 7-41 \pm 8$ & $25 \pm 7-42 \pm 4$ \\
\hline \multicolumn{4}{|l|}{ Apgar 1' } \\
\hline Média (DP) & $6,92(2,39)$ & $8,34(1,04)$ & $8,06(1,52)$ \\
\hline Mínimo-Máximo & $0-10$ & $0-10$ & $0-10$ \\
\hline \multicolumn{4}{|l|}{ Apgar 5' } \\
\hline Média (DP) & $8,66(1,31)$ & $9,34(, 59)$ & $9,20(, 83)$ \\
\hline Mínimo-Máximo & $2-10$ & $5-10$ & $2-10$ \\
\hline
\end{tabular}

n: número total; DP: desvio padrão. IRDA: indicadores de risco para a deficiência auditiva; Apgar 1': Apgar médio no primeiro minuto; Apgar 5': Apgar médio no quinto minuto

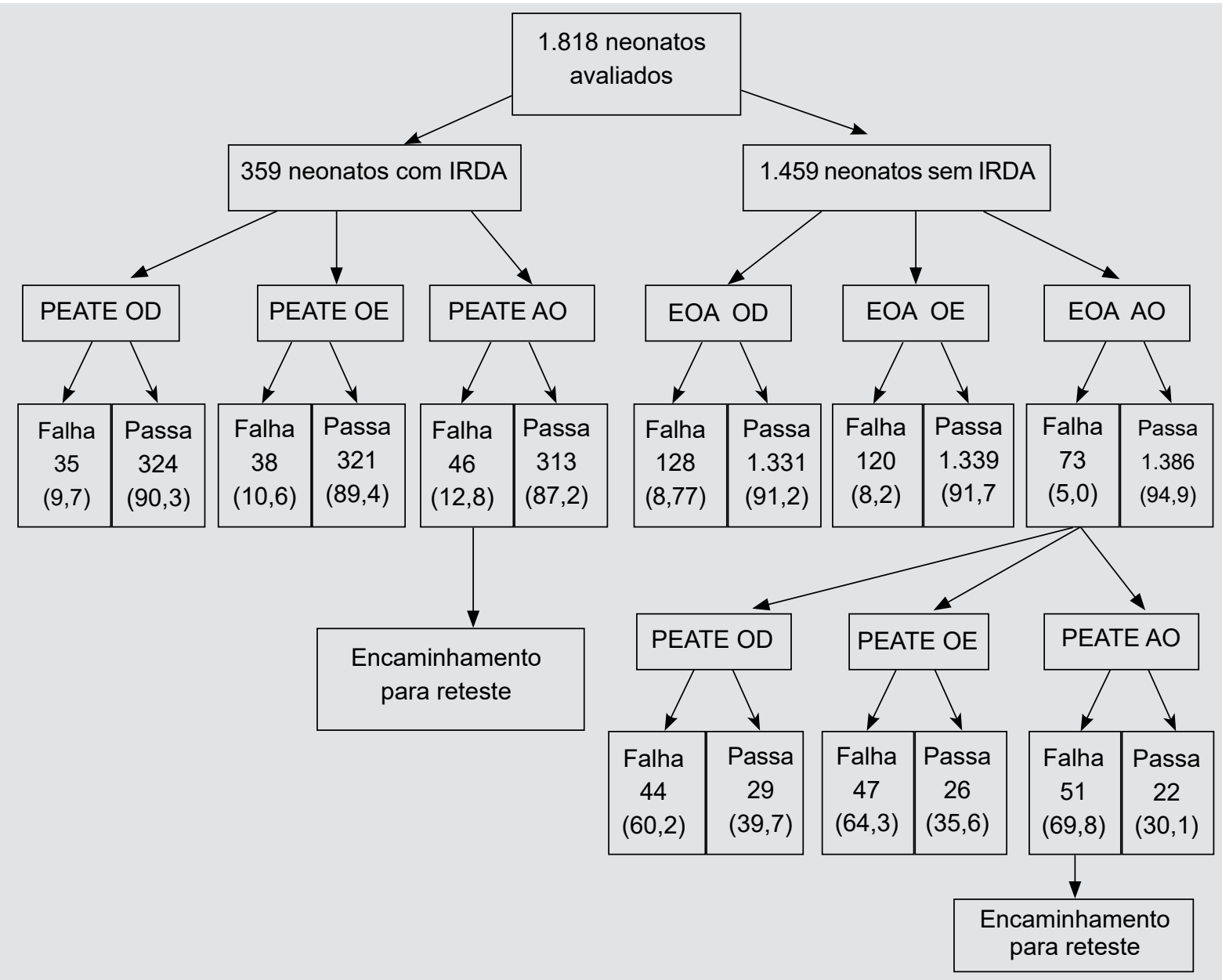

Figura 1 - Fluxograma da triagem auditiva neonatal dos neonatos nascidos entre maio de 2014 e maio de 2015, Hospital Materno-Infantil Presidente Vargas, Rio Grande do Sul, Brasil, 2016.

Legenda: OD: orelha direita; OE: orelha esquerda; AO: ambas as orelhas; PEATE: potencial evocado auditivo de tronco encefálico; EOA: emissões otoacústicas. Consideram-se os resultados numéricos como n (\%) 
Dentre os IRDA já estabelecidos na literatura, selecionaram-se para análise os resultados da TAN com exposição a drogas ototóxicas (antibióticos aminoglicosídeos e/ou diuréticos de alça pelo neonato), a infecções congênitas (toxoplasmose, rubéola, citomegalovírus, sífilis e HIV), consanguinidade, hereditariedade e síndrome que expressa deficiência auditiva no neonato, por serem mais prevalentes na amostra. Houve significância estatística nas falhas do PEATE em ambas as orelhas (AO) para aqueles com histórico de exposição ao HIV $(n=11 ; 22,9 \% ; p=0,035)$ e citomegalovírus ( $n=3 ; 42,9 ; p=0,048)$ congênitos. Embora tenham sido identificados hábitos e patologias maternas frequentes nas internações, nenhuma delas esteve associada à falha no PEATE em AO neste grupo. Identificaram-se, também, patologias referentes aos neonatos, sendo a disfunção respiratória (DR) $(n=9 ; 7,5 \% ; p=0,043)$ e a sepse $(n=6 ; 6,1 \% ; p=0,021)$ estatisticamente associadas à falha no PEATE em AO, como pode ser observado na Tabela II.

Na Tabela III, pode-se observar significância estatística nas falhas das EOA em AO em neonatos cujas mães tiveram infecção de trato urinário na gestação $(n=12 ; 6,6 \% ; p=0,015)$.

Tabela II - Resultados do potencial evocado auditivo de tronco encefálico comparados com indicadores de risco para deficiência auditiva e características maternas e do bebê, de neonatos que realizaram a triagem auditiva neonatal no Hospital Materno-Infantil Presidente Vargas, entre maio de 2014 e maio de 2015, Rio Grande do Sul, Brasil, 2016.

\begin{tabular}{|c|c|c|c|c|c|c|c|c|c|}
\hline & \multicolumn{3}{|c|}{ PEATE OE } & \multicolumn{3}{|c|}{ PEATE OD } & \multicolumn{3}{|c|}{ PEATE AO } \\
\hline & $\begin{array}{c}\text { Passa } \\
\text { n (\%) }\end{array}$ & $\begin{array}{l}\text { Falha } \\
\text { n (\%) }\end{array}$ & p valor & $\begin{array}{c}\text { Passa } \\
\text { n (\%) }\end{array}$ & $\begin{array}{l}\text { Falha } \\
\text { n (\%) }\end{array}$ & p valor & $\begin{array}{c}\text { Passa } \\
\text { n (\%) }\end{array}$ & $\begin{array}{l}\text { Falha } \\
\mathrm{n}(\%)\end{array}$ & p valor \\
\hline \multicolumn{10}{|l|}{ IRDA } \\
\hline Sífilis & $51(92,7)$ & $4(7,3)$ & 0,481 & $51(92,7)$ & $4(7,3)$ & 0,627 & $50(90,9)$ & $5(9,1)$ & 0,511 \\
\hline HIV & $41(85,4)$ & $7(14,6)$ & 0,318 & $38(79,2)$ & $\begin{array}{c}10 \\
(20,8)\end{array}$ & $0,015^{*}$ & $37(77,1)$ & $11(22,9)$ & $0,035^{*}$ \\
\hline Citomegalovírus & $5(71,4)$ & $2(28,6)$ & 0,163 & $5(71,4)$ & $2(28,6)$ & 0,142 & $4(57,1)$ & $3(42,9)$ & $0,048^{*}$ \\
\hline Toxoplasmose & $22(84,6)$ & $4(15,4)$ & 0,502 & $24(92,3)$ & $2(7,7)$ & 1,000 & $22(84,6)$ & $4(15,4)$ & 0,759 \\
\hline Consanguinidade & $2(66,7)$ & $1(33,3)$ & 0,286 & $2(66,7)$ & $1(33,3)$ & 0,266 & $2(66,7)$ & $1(33,3)$ & 0,338 \\
\hline Hereditariedade & $15(78,9)$ & $4(21,1)$ & 0,129 & $17(89,5)$ & $2(10,5)$ & 0,707 & $15(78,9)$ & $4(21,1)$ & 0,284 \\
\hline $\begin{array}{l}\text { Síndrome que expressa } \\
\text { deficiência auditiva no } \\
\text { neonato }\end{array}$ & $8(88,9)$ & $1(11,1)$ & 1,000 & $8(88,9)$ & $1(11,1)$ & 0,607 & $8(88,9)$ & $1(11,1)$ & 1,000 \\
\hline Exposição a drogas ototóxicas & $146(93,6)$ & $10(6,4)$ & $0,025^{*}$ & $149(95,5)$ & $7(4,5)$ & $0,004^{*}$ & $144(92,3)$ & $12(7,7)$ & $0,011^{*}$ \\
\hline \multicolumn{10}{|c|}{ Fatores maternos } \\
\hline Tabagista & $26(92,9)$ & $2(7,1)$ & 0,753 & $27(96,4)$ & $1(3,6)$ & 0,501 & $26(92,9)$ & $2(7,1)$ & 0,555 \\
\hline Drogadição & $32(88,9)$ & $4(11,1)$ & 0,782 & $31(86,1)$ & $5(13,9)$ & 0,374 & $30(83,3)$ & $6(16,7)$ & 0,435 \\
\hline ITU & $23(85,2)$ & $4(14,8)$ & 0,510 & $25(92,6)$ & $2(7,4)$ & 1,000 & $23(85,2)$ & $4(14,8)$ & 0,764 \\
\hline DMG & $26(86,7)$ & $4(13,3)$ & 0,541 & $27(90,0)$ & $3(10,0)$ & 1,000 & $26(86,7)$ & $4(13,3)$ & 1,000 \\
\hline HAS & $7(87,5)$ & $1(12,5)$ & 0,595 & $6(75,0)$ & $2(25,0)$ & 0,178 & $6(75,0)$ & $2(25,0)$ & 0,273 \\
\hline Hipotireoidismo & $7(77,8)$ & $2(22,2)$ & 0,245 & $8(88,9)$ & $1(11,1)$ & 0,607 & $7(77,8)$ & $2(22,2)$ & 0,324 \\
\hline Distúrbios psicológicos & $2(66,7)$ & $1(33,3)$ & 0,286 & $3(100)$ & $0(0)$ & 1,000 & $2(66,7)$ & $1(33,3)$ & 0,338 \\
\hline \multicolumn{10}{|l|}{ Fatores do neonato } \\
\hline DR & $113(94,2)$ & $7(5,8)$ & $0,045^{*}$ & $115(95,8)$ & $5(4,2)$ & $0,013^{*}$ & $111(92,5)$ & $9(7,5)$ & $0,043^{*}$ \\
\hline Icterícia & $81(91,0)$ & $8(9,0)$ & 0,693 & $83(93,3)$ & $6(6,7)$ & 0,310 & $80(89,9)$ & $9(10,1)$ & 0,466 \\
\hline Sepse & $94(94,9)$ & $5(5,1)$ & $0,036^{*}$ & $96(97,0)$ & $3(3,0)$ & $0,009^{*}$ & $93(93,9)$ & $6(6,1)$ & $0,021^{*}$ \\
\hline
\end{tabular}

OD: orelha direita; OE: orelha esquerda; AO: ambas orelhas; PEATE: potencial evocado auditivo de tronco encefálico; DR: disfunção respiratória; DMG: diabetes mellitus gestacional; HAS: hipertensão arterial sistêmica; ITU: infecção de trato urinário. *Houve significância estatística. O nível de significância máximo assumido foi de $5 \%(p \leq 0,05)$. A análise estatística destes dados foi realizada através do teste qui-quadrado de Pearson 
Tabela III - Resultados das emissões otoacústicas comparadas com características maternas e do bebê, de neonatos sem indicador de risco para deficiência auditiva que realizaram a triagem auditiva neonatal no Hospital Materno-Infantil Presidente Vargas, entre maio de 2014 e maio de 2015, Rio Grande do Sul, Brasil, 2016.

\begin{tabular}{lccccccccc}
\hline & \multicolumn{3}{c}{ EOA OE } & \multicolumn{3}{c}{ EOA OD } & & EOA AO \\
\cline { 2 - 9 } & $\begin{array}{c}\text { Passa } \\
\mathrm{n}(\%)\end{array}$ & $\begin{array}{c}\text { Falha } \\
\mathrm{n}(\%)\end{array}$ & $\mathrm{p}$ valor & $\begin{array}{c}\text { Passa } \\
\mathrm{n}(\%)\end{array}$ & $\begin{array}{c}\text { Falha } \\
\mathrm{n}(\%)\end{array}$ & $\mathrm{p}$ valor & $\begin{array}{c}\text { Passa } \\
\mathrm{n}(\%)\end{array}$ & $\begin{array}{c}\text { Falha } \\
\mathrm{n}(\%)\end{array}$ & $\mathrm{p}$ valor \\
\hline Fatores maternos & & & & & & & & & \\
$\quad$ Alcoolista & $6(100)$ & $0(0)$ & 1,000 & $6(100)$ & $0(0)$ & 1,000 & $6(100)$ & $0(0)$ & 1,000 \\
$\quad$ Tabagista & $121(89,0)$ & $15(11,0)$ & 0,249 & $118(86,8)$ & $18(13,2)$ & 0,078 & $116(85,3)$ & $20(14,7)$ & 0,331 \\
Drogadição & $22(91,7)$ & $2(8,3)$ & 1,000 & $19(79,2)$ & $5(20,8)$ & 0,052 & $19(79,2)$ & $5(20,8)$ & 0,196 \\
ITU & $175(95,6)$ & $8(4,4)$ & $0,043^{*}$ & $176(96,2)$ & $7(3,8)$ & $0,011^{*}$ & $171(93,4)$ & $12(6,6)$ & $0,015^{*}$ \\
DMG & $144(92,9)$ & $11(7,1)$ & 0,757 & $145(93,5)$ & $10(6,5)$ & 0,366 & $140(90,3)$ & $15(9,7)$ & 0,432 \\
HAS & $43(93,5)$ & $3(6,5)$ & 1,000 & $45(97,8)$ & $1(2,2)$ & 0,177 & $42(91,3)$ & $4(8,7)$ & 0,646 \\
Hipotireoidismo & $31(96,9)$ & $1(3,1)$ & 0,511 & $32(100)$ & $0(0)$ & 0,106 & $31(96,9)$ & $1(3,1)$ & 0,166 \\
Distúrbios psicológicos & $20(87,0)$ & $3(13,0)$ & 0,429 & $19(82,6)$ & $4(17,4)$ & 0,136 & $18(78,3)$ & $5(21,7)$ & 0,183 \\
Fatores do neonato & & & & & & & & & \\
DR & $29(100)$ & $0(0)$ & 0,164 & $29(100)$ & $0(0)$ & 0,171 & $29(100)$ & $0(0)$ & 0,041 \\
Icterícia & $38(100)$ & $0(0)$ & 0,068 & $38(100)$ & $0(0)$ & 0,072 & $38(100)$ & $0(0)$ & 0,011 \\
\hline
\end{tabular}

OD: orelha direita; OE: orelha esquerda; AO: ambas as orelhas; EOA: emissões otoacústicas; DR: disfunção respiratória; DMG: diabetes mellitus gestacional; HAS: hipertensão arterial sistêmica; ITU: infecção de trato urinário. *Houve significância estatística. O nível de significância máximo assumido foi de $5 \%(p \leq 0,05)$. A análise estatística destes dados foi realizada através do teste qui-quadrado de Pearson

\section{DISCUSSÃO}

Neste estudo analisaram-se dados de uma amostra de conveniência de um hospital materno-infantil da cidade de Porto Alegre, Rio Grande do Sul. Pôde-se notar maior prevalência do parto vaginal em comparação ao cesáreo. Esse achado pode ser decorrente de políticas públicas, como a RC e a Rede Humanizasus ${ }^{(1,9)}$, uma vez que o hospital em questão está vinculado a essas estratégias. De fato, comparando os achados do presente estudo com a média de parto vaginal na cidade de Porto Alegre $(48,11 \%)$, na Região Sul $(37,53 \%)$ e no Brasil $(42,85 \%)$, nota-se que o incentivo sobre a humanização do parto parece estar sendo efetivo, constituindo-se numa motivação para a realização de partos vaginais em maior número a mudança de concepção proposta pela RC e demais políticas materno-infantis.

No que diz respeito à Atenção Integral à Saúde da Criança, a deficiência auditiva é considerada um problema de saúde pública, em virtude de sua prevalência, e, sobretudo, pelas múltiplas consequências que pode acarretar ao desenvolvimento humano, nos aspectos intelectuais, sociais, linguísticos, cognitivos e emocionais ${ }^{(10)}$. Sendo assim, o controle e a análise dos dados epidemiológicos da TAN devem ser realizados por todos os fonoaudiólogos atuantes nessa atividade. Essas medidas são necessárias, tendo em vista o acompanhamento das mudanças na saúde populacional, que podem resultar em alterações e cuidados para a audição dessas crianças. Nesse sentido, chamam atenção as associações encontradas no presente estudo entre resultados da TAN e algumas características de saúde materna e infantil não consideradas nos IRDA, tais como infecção de trato urinário materno, sepse e disfunção respiratória. Dos fatores não associados aos IRDA, no grupo com pelo menos um indicador de risco, encontrou-se associação entre falha na TAN e sepse $(p=0,021)$. A sepse neonatal é a terceira principal causa de mortalidade neonatal, sendo superada apenas pela prematuridade e pelas complicações intraparto (ou asfixia no nascimento $)^{(11)}$. O tratamento empírico da sepse neonatal usualmente envolve associação entre um antibiótico aminoglicosídeo (gentamicina) e um betalactâmico, devendo-se, sempre, considerar o perfil ${ }^{(12)}$.

Também a capacidade respiratória do recém-nascido para se adaptar ao ambiente extrauterino é crítica para a sobrevivência. As disfunções respiratórias neonatais podem surgir por várias razões: adaptação atrasada ou má adaptação para a vida extrauterina; condições já existentes (anomalias congênitas ou cirúrgicas) ou adquiridas (infecções pulmonares ocorridas pré ou pós-parto) ${ }^{(13)}$.

Ainda nesse sentido, observou-se associação entre falha do bebê no teste de EOA e infecção de trato urinário materno $(p=0,015)$ e icterícia no próprio neonato $(p=0,011)$. A infecção do trato urinário é comum em mulheres jovens e representa uma das complicações clínicas mais frequentes no período gestacional. A gravidez ocasiona mudanças anatômicas e hormonais capazes de ter repercussão clínica, por exemplo: o aumento uterino gradual pode 
levar a algum grau de compressão extrínseca de via urinária, favorecendo o refluxo vesico-ureteral e aumentando a incidência de pielonefrite. Além disso, modificações da flora vaginal podem ocasionar vulvovaginites e infecções urinárias baixas (cistites). Se não tratadas, as mulheres podem sofrer complicações como trabalho de parto prematuro e restrição do crescimento intrauterino. O tratamento indicado para essas infecções de via urinária, usualmente, é a antibioticoterapia, entretanto os que são comumente utilizados não são ototóxicos ${ }^{(14)}$.

No que tange à icterícia, quando os bebês apresentam altos níveis séricos de bilirrubina (hiperbilirrubinemia), essa condição é considerada um IRDA. Adotou-se no hospital sob investigação o IRDA, neste caso, estabelecido pelo Joint committee on infant hearing ${ }^{(6)}$, hiperbilirrubinemia que exija transfusão exsanguínea ou cultura positiva para septicemia. Sendo assim, nessa amostra não foi identificado nenhum caso para realização do PEATE. Com esses resultados, faz-se necessária a instituição de protocolos com rigoroso controle dos casos de icterícia, bem como utilização de fototerapia altamente eficaz, que representam medidas de prevenção para as alterações auditivas decorrentes da hiperbilirrubinemia(15).

A TAN, no presente estudo, foi realizada nas primeiras horas de vida do neonato, antes da alta hospitalar, e conforme o fluxograma e as técnicas propostas pelo Ministério da Saúde (2012) ${ }^{(5)}$. Considerando os IRDA previstos, as infecções congênitas (HIV e citomegalovírus) e a exposição a drogas ototóxicas (antibióticos aminoglicosídeos e/ou diuréticos de alça) pelo neonato foram fatores associados com a falha no PEATE. Esses achados, portanto, concordam com a literatura ${ }^{(7)}$.

A exposição ao HIV intraútero mostrou-se como a segunda infecção congênita mais prevalente na atual pesquisa $(n=48)$, sendo superada apenas pela sífilis $(n=55)$, e estando associada à falha na TAN ( $p=0,035)$. A associação entre infecção pelo HIV e deficiência auditiva está sendo relatada na literatura e o que tem sido visto é que, na fase inicial da doença, o acometimento acaba sendo menor e, com o passar dos anos, as desordens se tornam mais frequentes ${ }^{(16)}$. As manifestações clínicas são muito variáveis, podendo ser evidenciadas por: encefalopatias, tanto de natureza estática quanto de natureza progressiva; retardo do desenvolvimento neuropsicomotor; atraso de linguagem; deficiência mental; hiporreflexia; e sinais piramidais ${ }^{(17)}$. Dentre as inúmeras infecções que podem acometer crianças com HIV, estão as que afetam as vias aéreas superiores, principalmente as sinusites e as otites externa e média, responsáveis pela alta incidência de alterações auditivas condutivas. Assim, nessas crianças, o comprometimento da audição pode ter relação com outros fatores, entre eles, a própria ação lesiva viral sobre as estruturas nervosas periféricas e centrais. Portanto, o HIV pode ser considerado um fator de risco para alteração auditiva tanto periférica quanto central para crianças e adolescentes ${ }^{(18,19)}$.

Já a exposição ao citomegalovírus (CMV) foi pouco frequente na amostra $(n=7)$ da presente pesquisa, estando, mesmo assim, associada à falha na TAN $(p=0,048)$. O CMV pertence à família dos herpes vírus e é causa frequente de infecção nos seres humanos. Esse vírus sofre períodos de ativação e latência e, uma vez infectado, o vírus permanece indefinidamente no corpo do hospedeiro, podendo haver uma reativação a qualquer momento ${ }^{(20,21)}$. A transmissão vertical do CMV pode ser transplacentária após uma infeç̧ão materna primária ou recorrente, mas também pode ocorrer se houver exposição às secreções contaminadas do trato genital inferior (TGI) no momento do parto ou durante a amamentação( ${ }^{(21)}$. Esses neonatos podem ter comprometimento cognitivo, retinite, e/ou paralisia cerebral, além da perda auditiva sensorioneural, considerada uma das sequelas mais comuns, o que explica os resultados do presente estudo. A perda auditiva após infecção congênita por CMV pode ter início tardio, embora perdas progressivas possam ocorrer. Sendo assim, as crianças com infecção por CMV devem ser avaliadas regularmente para assegurar a detecção precoce da perda auditiva e seu adequado atendimento ${ }^{(22)}$.

Portanto, reforça-se que a promoção da saúde se relaciona a esses conceitos, uma vez que a realização da TAN, bem como a saúde geral da população, é fator influenciador. É importante deixar a população capacitada para atuar na sua qualidade de vida, incentivando que ela tenha o controle e saiba a importância desse processo. Esse conhecimento poderá qualificar a assistência já prestada no âmbito da RC ao possibilitar a elaboração de novas ações e estratégias de atenção ao RN e suas mães, não apenas pelos profissionais da Fonoaudiologia, mas também por todos aqueles que integram a equipe de assistência.

Como limitação deste estudo, pode-se apontar a regionalização dos dados, pois traz resultados de uma amostra populacional de um hospital maternoinfantil, podendo esses resultados diferirem em outros locais do Brasil. Sendo assim, são necessários novos estudos para descrever as características e resultados da TAN nas demais regiões do Brasil.

Considera-se que a produção de dados epidemiológicos sobre a TAN deve ser estimulada, para que haja fundamentação nos elementos causais e para que sirva de base para o desenvolvimento de estratégias e políticas públicas de prevenção e promoção de saúde auditiva. 


\section{CONCLUSÃO}

Neste estudo foi possível reconhecer as associações da falha na triagem auditiva neonatal com indicadores de risco para deficiência auditiva decorrentes de doenças infectocontagiosas (HIV e CMV) e exposição às drogas ototóxicas. Além desses, houve também associação com icterícia neonatal, disfunção respiratória, sepse e infecção de trato urinário materno. O encaminhamento para o diagnóstico, a interpretação de como a população desse hospital está se comportando e a possibilidade de comparar esses dados com outros hospitais ajudam a nortear as intervenções em saúde, visando à redução de riscos de doenças e de outros agravos e o estabelecimento de condições que assegurem aos serviços possibilidades para atuar na promoção, proteção e recuperação em saúde.

\section{CONFLITOS DE INTERESSE}

As autoras afirmam que não houve conflitos de interesses na execução desta pesquisa.

\section{CONTRIBUIÇÕES}

Luíza Silva Vernier, Carla Castelli e Shayenne Rothermel contribuíram com a concepção do artigo, a coleta dos dados, a análise e discussão dos dados e a redação do artigo. Tatiana Paniz e Claudia Zanini contribuíram com a concepção do artigo e a análise e discussão dos dados. Daniela Centenaro Levandowski contribuiu com a supervisão de todas as etapas de elaboração do artigo, incluindo a análise e discussão dos dados e a redação do artigo.

\section{REFERÊNCIAS}

1. Brasil Ministério da Saúde. Secretaria de Atenção à Saúde. Departamento de Ações Programáticas Estratégicas. Portaria no 1.459, 24 de junho de 2011. Institui, no âmbito do Sistema Único de Saúde, a Rede Cegonha. Diário Oficial da União, 24 de junho de 2012.

2. Sabbag JC, Lacerda ABM. Rastreamento e monitoramento da Triagem Auditiva Neonatal em Unidade de Estratégia de Saúde da Família: estudo-piloto. CoDAS. 2017;29:4.

3. Brasil. Lei $n^{\circ} 12.303$, de 02 de agosto de 2010. Dispõe sobre a obrigatoriedade de realização do exame denominado Emissões Otoacústicas Evocadas. Diário Oficialda União, 03 de agosto de 2010.

4. Silva DPC, Lopez OS, Montovani JC. Influência dos indicadores de risco nas diferentes etapas da Triagem Auditiva Neonatal. Audiol Commun Res. 2015;21:e1614.

5. Ministério da Saúde (BR). Secretaria de Atenção à Saúde. Departamento de Ações Programáticas Estratégicas. Diretrizes de Atenção da Triagem Auditiva Neonatal. Brasília: Ministério da Saúde; 2012.

6. Joint Committee on Infant Hearing. Ano 2007 declaração de posição: princípios e orientações para os programas de detecção e intervenção auditiva precoce. Pediatrics. 2007;120:898-921.

7. Lewis DR, Marone SAM, Mendes BCA, Cruz OLM, Nóbrega M. Comitê multiprofissional em saúde auditiva: COMUSA. Braz J Otorhinolaryngol. 2010;76:121-28.

8. Conselho Nacional de Saúde (BR). Resolução no 466, de 12 de dezembro de 2012. Brasília: Conselho Nacional de Saúde; 2012.

9. Ministério da Saúde (BR). Secretaria de Atenção à Saúde. Núcleo Técnico da Política Nacional de Humanização. Cadernos HumanizaSUS: Humanização do parto e nascimento. Brasília: Ministério da Saúde; 2014

10. Kemp AAT, Delecrode CR, Silva GC, Martins F, Frizzo ACF, Cardoso ACV. Neonatal hearing screening in a low-risk maternity hospital in São Paulo state. Braz J Otorhinolaryngol. 2015;81:505-13.

11. Zea-Vera A, Ochoa TJ. Desafios no diagnóstico e tratamento da sepse neonatal. J Trop Pediatr. 2015;61:1-13.

12. Miura E, Silveira RC, Procianoy RS. Sepse neonatal: diagnóstico e tratamento. J Pediatr (Rio J). 1999;75:5762. 
13. Gallacher DJ, Hart K, Kotecha S. Common respiratory conditions of the newborn. Breathe (Sheff). 2016;12(1):30-42.

14. Carvalho $\mathrm{Cl}$. Infecção do trato urinário associado a gestantes e o papel do profissional farmacêutico no tratamento farmacoterapêutico. FACIDER Rev Cient. 2015;4(7):1-18.

15. Lima GML, Marba STM, Santos MFC. Hearing screening in a neonatal intensive care unit. J Pediatr. 2006;82:110-4.

16. Padilha MAD, Maruta ECS, Azevedo MF. Occurrence of hearing disorders in infants exposed to HIV vertical transmission. Audiol Commun Res. 2018;23:e1965

17. Guedes-Granzotti RB, Fukuda MTH, Silva KD, Dornelas R, Domenis DR, Takayanagui OM. Oral and written language of children seropositive for HIV: a longitudinal follow up. Audiol Commun Res. 2017;22:e1852.

18. Sousa TKS. Avaliação audiológica de crianças vivendo com HIV: revisão de literatura [trabalho de conclusão de curso]. São Luís: UFMA; 2017.

19. Torre P, Zeldow B, Yao TJ, Hoffman HJ, Siberry GK, Purswani MU, et al. Newborn Hearing Screenings in Human Immunodeficiency Virus-Exposed Uninfected Infants. J AIDS Immune Res. 2016;1(1):1-10.

20. Borges FPS, Souza MBLD. Citomegalovírus em pacientes submetidos a transplante de células progenitoras hematopoiéticas [dissertação]. Goiânia: Universidade Federal de Goiás; 2016.

21. Núcleo de Telessaúde Sergipe. Qual o papel da enfermagem diante do resultado de citomegalovírus IgM reagente de gestantes [Internet]? 2017 [acesso em 2019 mar 15]. Disponível em: https://aps.bvs.br/aps/qualo-papel-da-enfermagem-diante-do-resultado-de-citomegalovirus-igm-reagente-de-gestantes/

22. Fowler KB. Congenital Cytomegalovirus Infection: audiologic outcome. Clin Infect Dis. 2013;57:182-84.

\author{
Endereço do primeiro autor: \\ Luíza Silva Vernier \\ Universidade Federal de Ciências da Saúde de Porto Alegre - UFCSPA \\ Rua Sarmento Leite, 245/sala 207 \\ Bairro: Centro Histórico. \\ CEP: 90050-170 - Porto Alegre - RS - Brasil \\ E-mail: luiza.vernier@hotmail.com

\section{Endereço para correspondência:} \\ Daniela Centenaro Levandowski \\ Universidade Federal de Ciências da Saúde de Porto Alegre - UFCSPA \\ Rua Sarmento Leite, 245/sala 207 \\ Bairro: Centro Histórico. \\ CEP: 90050-170 - Porto Alegre - RS - Brasil \\ E-mail: daniela@ufcspa.edu.br
}

Como citar: Vernier LS, Castelli CTR, Rothermel SS, Paniz TC, Zanini C, Levandowski DC. Triagem auditiva neonatal em hospital da rede cegonha. Rev Bras Promoç Saúde. 2019;32:8965. 